\title{
Lyssavirus in Japanese Pipistrelle, Taiwan
}

\author{
Shu-Chia Hu, Chao-Lung Hsu, Ming-Shiuh Lee, \\ Yang-Chang Tu, Jen-Chieh Chang, \\ Chieh-Hao Wu, Shu-Hwae Lee, Lu-Jen Ting, \\ Kwok-Rong Tsai, Ming-Chu Cheng, \\ Wen-Jane Tu, Wei-Cheng Hsu
}

A putative new lyssavirus was found in 2 Japanese pipistrelles (Pipistrellus abramus) in Taiwan in 2016 and 2017. The concatenated coding regions of the virus showed $62.9 \%-75.1 \%$ nucleotide identities to the other 16 species of lyssavirus, suggesting that it may be representative of a new species of this virus.

$\mathrm{T}$ he Lyssavirus genus within the family Rhabdoviridae is composed of 14 species of lyssavirus: rabies lyssavirus (RABV), Lagos bat lyssavirus (LBV), Mokola lyssavirus (MOKV), Duvenhage lyssavirus (DUVV), European bat 1 lyssavirus (EBLV-1), European bat 2 lyssavirus (EBLV-2), Australian bat lyssavirus (ABLV), Aravan lyssavirus (ARAV), Khujand lyssavirus (KHUV), Irkut lyssavirus (IRKV), Shimoni bat lyssavirus (SHIBV), Bokeloh bat lyssavirus (BBLV), West Caucasian bat lyssavirus (WCBV), and Ikoma lyssavirus (IKOV) (1). In addition, Lleida bat lyssavirus (LLEBV) $(1,2)$ and Gannoruwa bat lyssavirus (GBLV) (3) were recently identified in bats, but their taxonomic statuses have not been determined by the International Committee on the Taxonomy of Viruses. The genus Lyssavirus can be subdivided into phylogroup 1 (RABV, DUVV, EBLV-1, EBLV-2, ABLV, ARAV, KHUV, IRKV, BBLV, and GBLV) and phylogroup 2 (LBV, MOKV, and SHIBV) according to genetic distances and serologic cross-reactivity (1-3). The remaining species, WCBV, IKOV, and LLEBV, cannot be included in either of these phylogroups $(1,2)$.

Bats are the natural hosts of most lyssaviruses, with the exceptions of MOKV and IKOV, which have not been identified in any bats (1-4). Information about lyssaviruses in bats in Asia is limited. In Central Asia, ARAV was

Author affiliations: Animal Health Research Institute, New Taipei

City, Taiwan (S.-C. Hu, M.-S. Lee, Y.-C. Tu, J.-C. Chang,

C.-H. Wu, L.-J. Ting, K.-R. Tsai, W.-J. Tu, W.-C. Hsu); National

Taiwan University, Taipei City, Taiwan (C.-L. Hsu); Bat

Conservation Society of Taipei, Taipei City (C.-L. Hsu); Animal

Health Research Institute, Miaoli County, Taiwan (S.-H. Lee);

National Pingtung University of Science and Technology, Neipu

Township, Pingtung County, Taiwan (M.-C. Cheng)

DOI: https://doi.org/10.3201/eid2404.171696 identified in the lesser mouse-eared bat (Myotis blythi) in Kyrgyzstan in 1991, and KHUV was identified in the whiskered bat (M. mystacinus) in Tajikistan in 2001 (5). In South Asia, GBLV was identified in the Indian flying fox (Pteropus medius) in Sri Lanka in 2015 (3). Although IRKV was identified in the greater tube-nosed bat (Murina leucogaster) in China in 2012 (6), knowledge of the exact species and locations of lyssaviruses in East Asia bat populations remains limited.

In this article, we report a putative new lyssavirus isolated during our surveillance program in Taiwan. Our discovery suggests that this lyssavirus may be representative of a new species, based on genetic distance.

\section{The Study}

Specimens for this study were collected under a permit issued by the Forestry Bureau, Council of Agriculture, Executive Yuan, Taiwan (document no. 1055104969). From 2014 through the end of May 2017, a total of 332 bat carcasses from 13 species were collected for lyssavirus surveillance. Of the collected individuals, 2 tested positive for the virus by direct fluorescent antibody testing and reverse transcription PCR (7-9). The first bat showing loss of appetite without specific clinical signs was found in Tainan City and died on July 2, 2016. The second bat was found dead in Yunlin County on April 12, 2017, and the carcass was shipped to the Animal Health Research Institute (AHRI) in New Taipei City. We obtained two 428-bp amplicons (N113F/N304R, containing the partial nucleoprotein $[\mathrm{N}]$ gene and phosphoprotein $[\mathrm{P}]$ gene) from these cases using lyssavirus screen primers (Table 1); we then subjected their sequences to BLAST (https://www.ncbi.nlm.nih.gov/ BLAST/), querying the GenBank database. Both sequences were similar to lyssaviruses, with nucleotide identities $<79 \%$. The 2 bats were identified as Japanese pipistrelle (Pipistrellus abramus), or Japanese house bat, by morphology (J.T. Wu, Taxonomic study of the genus Pipistrellus [chiroptera: vespertilionidae] in Taiwan. Master's thesis, Department of Biological Resources, National Chiayi University, Chiayi City, Taiwan, 2007 [in Chinese]) and DNA barcoding (based on subunit 1 of the mitochondrial protein NADH dehydrogenase [ND1] gene) (10). The 2 sequences of partial ND1 genes were then submitted to GenBank; the first had been designated as 2016-2300 (GenBank accession no. MG763889) and the second 2017-1502 (GenBank accession no. MG763890).

We isolated lyssavirus successfully from the 2 bats' brains; we confirmed the identity of lyssavirus 
Lyssavirus in Japanese Pipistrelle, Taiwan

Table 1. Lyssavirus screen primers and the 12 amplifying primer sets used to identify Taiwan bat lyssavirus, a putative new lyssavirus found in 2 Japanese pipistrelles (Pipistrellus abramus) in Taiwan in 2016 and 2017

\begin{tabular}{|c|c|c|}
\hline Primer name & Sequence, $5^{\prime} \rightarrow 3^{\prime}$ & Position* \\
\hline \multicolumn{3}{|l|}{ Lyssavirus screen } \\
\hline JW12 (7) & ATGTAACACCYCTACAATG & \\
\hline N165-146 (7) & GCAGGGTAYTTRTACTCATA & \\
\hline N113F (8) & GTAGGATGCTATATGGG & \\
\hline N304R (9) & TTGACGAAGATCTTGCTCAT & \\
\hline \multicolumn{3}{|c|}{ Lyssavirus full genome } \\
\hline TWBLV 1F & ACGCTTAACGACAAAAYC & $1-18^{*}$ \\
\hline TWBLV 1R & TCTTGCATTTCTTTCTCATC & $1154-1173$ \\
\hline TWBLV 2F & TTCGTAGGATGTTACATGGG & $1010-1029$ \\
\hline TWBLV 2R & TAAAAATATCCCAGAAGATC & 2174-2193 \\
\hline TWBLV 3F & AGARATAGCWCATCAGATWGC & $2118-2138$ \\
\hline TWBLV 3R & CTATTGTGTGGCACCATWAC & $3205-3224$ \\
\hline TWBLV 4F & GATGAGGATAAGAACACATC & $3072-3091$ \\
\hline TWBLV 4R & TCCTGAAGTGACTGAGTTTTC & $4274-4294$ \\
\hline TWBLV 5F & CTGATGGAYGGRTCATGGGT & $4081-4100$ \\
\hline TWBLV 5R & GAGACAGGAGCCGGAGTCTT & $5280-5299$ \\
\hline TWBLV 6F & AACAGGTAGCTCCCGAGTTTGTTC & $4881-4904$ \\
\hline TWBLV 6R & CTGAGTGAGACCCATGTATCCAAA & $5767-5790$ \\
\hline TWBLV 7F & ACTGAGGTTTATGATGACCC & $5478-5497$ \\
\hline TWBLV 7R & CCCCAGTGTCTATARCAWCC & $6561-6580$ \\
\hline TWBLV 8F & CATTCTTTGGGGGATTTCCC & $6426-6445$ \\
\hline TWBLV 8R & GTTTGTGATTCTCTRTCWATC & $7595-7615$ \\
\hline TWBLV 9F & CATGCTGGAACGGTCAGGAYG & $7522-7542$ \\
\hline TWBLV 9R & CTGAGTTAAAGAAAGATTCTT & 8662-8682 \\
\hline TWBLV 10F & CTCAGTGAGTTRTTYAGCTC & $8547-8566$ \\
\hline TWBLV 10R & CAGATAGAAGAGCCTATT & 9746-9763 \\
\hline TWBLV 11F & CATGATTCAGGGTAYAAYGA & 9648-9667 \\
\hline TWBLV 11R & GTCTGTAACTTCTGCATCAC & $10862-10871$ \\
\hline TWBLV 12F & ATCTGGGAAAAGCCATCAGA & $10755-10774$ \\
\hline TWBLV 12R & ACGCTTAACAAAAAAAACAA & $11961-11980$ \\
\hline
\end{tabular}

morphologically by electron microscopy (11) and molecularly by nucleotide sequencing. In addition, after we isolated the viruses from the brain tissues, we performed reverse transcription PCR and virus isolation for visceral organs and salivary glands; both tests were positive for the salivary glands of both bats.
We determined nucleotide sequences of the genome of the isolated viruses, designated Taiwan bat lyssavirus (TWBLV). The first TWBLV isolate was designated TWBLV/TN/2016 (GenBank accession no. MF472710), and the second TWBLV/YL/2017 (GenBank accession no. MF472709). We amplified the nucleoprotein (N),

Table 2. Nucleotide identities for the N, P, M, G, L genes and the concatenated coding genes between Taiwan bat lyssavirus, a putative new lyssavirus found in 2 Japanese pipistrelles (Pipistrellus abramus) in Taiwan in 2016 and 2017, compared with other lyssaviruses*

\begin{tabular}{|c|c|c|c|c|c|c|}
\hline \multirow[b]{2}{*}{ Lyssavirus species (GenBank accession no.) } & \multicolumn{5}{|c|}{ Identity, \% } & \multirow{2}{*}{$\begin{array}{l}\text { Concatenated } \\
\text { coding genes } \\
(\mathrm{N}+\mathrm{P}+\mathrm{M}+\mathrm{G}+\mathrm{L})\end{array}$} \\
\hline & $\mathrm{N}$ & $\mathrm{P}$ & $\mathrm{M}$ & $\mathrm{G}$ & $\mathrm{L}$ & \\
\hline Rabies lyssavirus (NC001542) & 75.2 & $62.8-63.1$ & $74.6-74.8$ & $61.6-62.3$ & 71.7 & $70.0-70.1$ \\
\hline Lagos bat lyssavirus (NC020807) & $73.5-73.7$ & $53.8-54.1$ & 73.6 & $55.0-55.6$ & $71.3-71.4$ & $67.8-67.9$ \\
\hline Mokola lyssavirus (NC006429) & $72.7-73.3$ & $52.2-52.4$ & $72.0-72.6$ & $56.7-56.9$ & $70.6-70.7$ & $67.3-67.4$ \\
\hline Duvenhage lyssavirus (NC020810) & $77.7-77.9$ & $67.5-67.6$ & $78.4-79.2$ & $63.9-64.0$ & $74.8-74.9$ & 73.2 \\
\hline European bat 1 lyssavirus (NC009527) & $78.8-79.4$ & $69.6-69.7$ & $80.9-81.1$ & 66.1 & $76.7-76.8$ & 75.1 \\
\hline European bat 2 lyssavirus (NC009528) & $75.3-75.6$ & $66.0-66.3$ & $79.2-79.5$ & $64.5-64.6$ & 73.7 & $72.2-72.3$ \\
\hline Australian bat lyssavirus (NC003243) & $76.3-76.5$ & 62.2 & 72.1 & $61.7-62.0$ & $72.3-72.5$ & 70.4 \\
\hline Aravan lyssavirus (NC020808) & $76.5-76.7$ & $65.6-66$ & $80.3-80.6$ & $64.4-64.5$ & $74.6-74.9$ & $72.9-73.1$ \\
\hline Khujand lyssavirus (NC025385) & $74.6-74.7$ & $67.0-67.1$ & $78.6-78.7$ & $64.5-64.9$ & $74.2-74.3$ & $72.4-72.5$ \\
\hline Irkut lyssavirus (NC020809) & $79.0-79.1$ & $68.3-68.6$ & 80.3 & $65.3-65.8$ & $75.3-75.4$ & 74 \\
\hline Irkut lyssavirus-THChina12 (JX442979) & $80.5-80.6$ & $68.7-69.0$ & $79.8-80.0$ & $64-64.6$ & $75.1-75.4$ & 74 \\
\hline West Caucasian bat lyssavirus (NC025377) & $72.3-72.7$ & $51.2-51.5$ & 71.4 & 52.0 & $68.3-68.4$ & $65-65.2$ \\
\hline Shimoni bat lyssavirus (NC025365) & $74.1-74.5$ & $54.9-55.1$ & $74.2-74.6$ & $56.2-56.4$ & $71.1-71.2$ & 68.2 \\
\hline Ikoma lyssavirus (NC018629) & $69.7-69.8$ & $51.9-52.3$ & $69.3-69.6$ & $50.9-51.2$ & $65.4-65.6$ & 62.9 \\
\hline Bokeloh bat lyssavirus (NC025251) & $75.0-75.2$ & $66.0-66.3$ & 78.2 & $64.4-64.5$ & $74.1-74.2$ & $72.3-72.4$ \\
\hline Lleida bat lyssavirus (NC031955) & $69.2-69.5$ & $50.8-50.9$ & $70.4-70.9$ & 51.2 & 66.2 & $63.2-63.3$ \\
\hline Gannoruwa bat lyssavirus (NC031988) & $75.5-75.7$ & $62.8-63.1$ & $75.4-75.7$ & $63.7-64.3$ & $73.5-73.6$ & $71.5-71.6$ \\
\hline
\end{tabular}


Figure. Phylogenetic relationship of TWBLV (boldface), a putative new lyssavirus found in 2 Japanese pipistrelles (Pipistrellus abramus) in Taiwan in 2016 and 2017, compared with other lyssaviruses. Using the concatenated coding genes, we constructed the phylogenetic tree by using the maximumlikelihood method with the general time reversible plus invariant sites plus gamma 4 model. Numbers at the nodes indicate bootstrap confidence values (1,000 replicates) for the groups being composed of virus genes at the right of the nodes. GenBank accession numbers are provided for reference viruses. Scale bar indicates nucleotide substitutions per site. ABLV, Australia bat lyssavirus; ARAV, Aravan lyssavirus; BBLV, Bokeloh bat lyssavirus; DUVV, Duvenhage lyssavirus; EBLV-1, European bat lyssavirus type 1; EBLV-2,

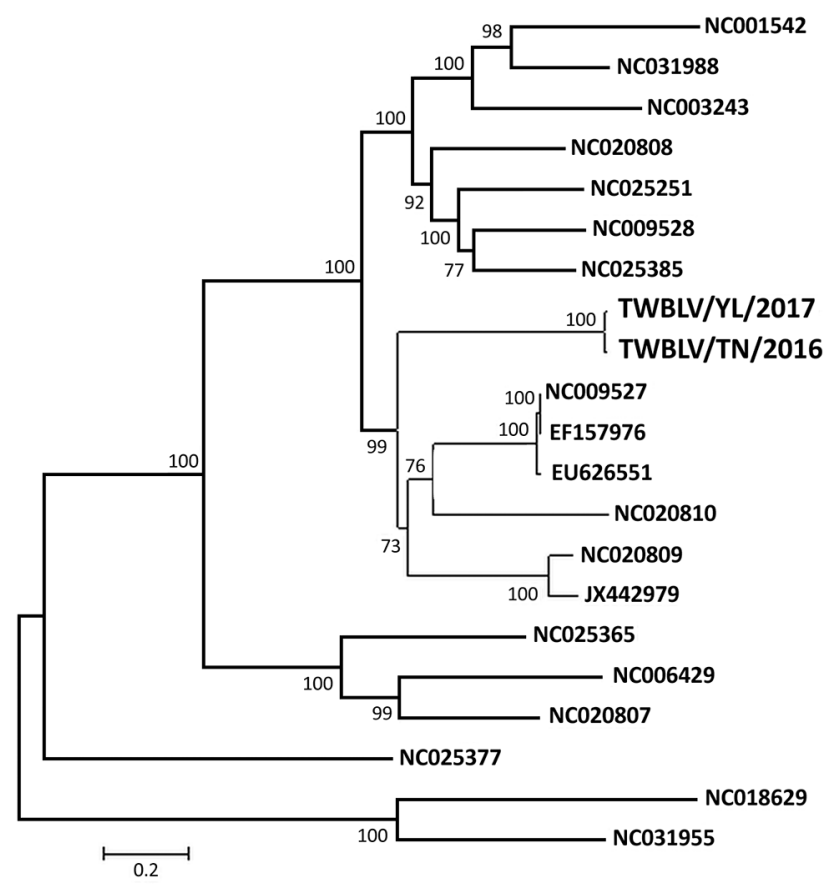

\begin{tabular}{l|l} 
I RABV & \\
I GBLV & \\
I ABLV & \\
I ARAV & \\
I BBLV & \\
I EBLV-2 & \\
I KHUV & Phylogroup 1 \\
& \\
I EBLV-1 & \\
I DUVV & \\
I IRKV & \\
I SHIBV & Phylogroup 2 \\
I MOKV & \\
I LBV & \\
I WCBV & \\
I IKOV & \\
I LLEBV &
\end{tabular}
European bat lyssavirus type

2; IKOV, Ikoma lyssavirus; IRKV, Irkut lyssavirus; KHUV, Khujand lyssavirus; LBV, Lagos bat lyssavirus; LLEBV, Lleida bat lyssavirus; MOKV, Mokola lyssavirus; SHIBV, Shimoni bat lyssavirus; RABV, rabies lyssavirus; TWBLV, Taiwan bat lyssavirus; WCBV, West Caucasian bat lyssavirus.

phosphoprotein $(\mathrm{P})$, matrix protein $(\mathrm{M})$, glycoprotein $(\mathrm{G})$, and RNA-dependent RNA polymerase (L) genes of the 2 TWBLV isolates by 12 primer sets designed by the AHRI (Table 1). The genome length of the 2 TWBLV isolates was 11,988 bases, with $\mathrm{G}+\mathrm{C}$ contents of $43.66 \%$ for TWBLV/ $\mathrm{TN} / 2016$ and $43.83 \%$ for TWBLV/YL/2017. The genomic organization was similar to those of other lyssaviruses: $3^{\prime}$ untranslated region (UTR), $70 \mathrm{nt}$; N, $1356 \mathrm{nt}$; N-P UTR, $99 \mathrm{nt}$; P, 897 nt; P-M UTR, 82 nt; M, 609 nt; M-G UTR, 212 nt; G,1629 nt; G-L UTR, 518 nt; L, 6384 nt; and 5' UTR, 132 nt. The 2 TWBLV isolates showed $98.7 \%$ nucleotide identity in the $\mathrm{N}$ gene and $98.6 \%$ nucleotide identity in the concatenated coding genes $(\mathrm{N}+\mathrm{P}+\mathrm{M}+\mathrm{G}+\mathrm{L})$. Nucleotide identities of different genes between the TWBLVs and the other 16 lyssaviruses are listed in Table 2. For the N gene, the TWBLVs had the highest identities with IRKV $(79.0 \%-80.6 \%)$, followed by EBLV-1 (78.8\%-79.4\%). The TWBLVs shared nucleotide identities with the concatenated coding genes of EBLV1 (75.1\%), followed by IRKV (74.0\%). The phylogenetic analysis demonstrated that lyssaviruses were separated into 2 phylogroups; the TWBLVs were grouped into phylogroup 1 and clustered with the EBLV-1, IRKV, and DUVV (Figure).

\section{Conclusions}

We report a lyssavirus, TWBLV, that is closely related to EBLV-1, IRKV, and DUVV. The full-length nucleotide sequence of the concatenated coding genes of TWBLV showed $62.9 \%-75.1 \%$ nucleotide identities to the other 16 lyssaviruses (Table 2), and TWBLV was the most closely related to EBLV-1. The demarcation criteria of lyssavirus species, established by the International Committee on the Taxonomy of Viruses, include genetic distance, topology, antigenic patterns, and additional characteristics (12). Based on genetic distance, no similarity to the other lyssavirus species of more than $75.1 \%$ nucleotide identities of the concatenated coding genes of TWBLVs suggested that the isolated TWBLV was a new lyssavirus species.

The presence of TWBLV in the bats' salivary glands suggested that TWBLV may be shed through saliva. The study showed that the bat in East Asia could be infected with lyssavirus; however, because of the limited surveillance, the epidemiology of lyssavirus in Japanese pipistrelle and other bat species is still unclear. This uncertainty is likely to raise a public health concern in countries in Asia.

In conclusion, a lyssavirus, TWBLV, was identified in Japanese pipistrelle, and the infected bats may shed the virus through saliva. Japanese pipistrelle is a common insectivorous bat of low-altitude urban areas in East Asia $(13,14)$. Persons in countries in Asia should be aware to seek proper prophylaxis immediately if bitten by a bat. Studies on the epidemiology and pathogenicity of TWBLV are necessary to further characterize the virus. 


\section{Acknowledgments}

We thank Fan Lee for his help in improving the manuscript. We also thank Tien-Cheng Li, Yi-Tang Lin, Chia-Jung Tsai, and Ya-Lan Li for their assistance during this study.

This study was supported by grant no. 106AS-9.9.1-BQ-B2(1) from the Bureau of Animal and Plant Health Inspection and Quarantine, Council of Agriculture, Executive Yuan, Taiwan.

\section{About the Author}

Dr. Hu is an assistant research fellow at Epidemiology Division, Animal Health Research Institute, New Taipei City, Taiwan. Her main research interests are in the molecular epidemiology of lyssaviruses.

\section{References}

1. Kuzmin IV. Basic facts about lyssavirus. In: Rupprecht CE, Nagarajan T, editor. Current laboratory techniques in rabies diagnosis, research, and prevention, volume 1. Laguna Hills (CA): Elsevier; 2014. p. 3-17.

2. Aréchiga Ceballos N, Vázquez Morón S, Berciano JM, Nicolás O, Aznar López C, Juste J, et al. Novel lyssavirus in bat, Spain. Emerg Infect Dis. 2013;19:793-5. http://dx.doi.org/10.3201/ eid1905.121071

3. Gunawardena PS, Marston DA, Ellis RJ, Wise EL, Karawita AC, Breed AC, et al. Lyssavirus in Indian flying foxes, Sri Lanka. Emerg Infect Dis. 2016;22:1456-9. http://dx.doi.org/10.3201/ eid2208.151986

4. Banyard AC, Evans JS, Luo TR, Fooks AR. Lyssaviruses and bats: emergence and zoonotic threat. Viruses. 2014;6:2974-90. http://dx.doi.org/10.3390/v6082974

5. Kuzmin IV, Orciari LA, Arai YT, Smith JS, Hanlon CA, Kameoka Y, et al. Bat lyssaviruses (Aravan and Khujand) from Central Asia: phylogenetic relationships according to N,
P and G gene sequences. Virus Res. 2003;97:65-79.

http://dx.doi.org/10.1016/S0168-1702(03)00217-X

6. Liu Y, Zhang S, Zhao J, Zhang F, Hu R. Isolation of Irkut virus from a Murina leucogaster bat in China. PLoS Negl Trop Dis. 2013; 7:e2097. http://dx.doi.org/10.1371/journal.pntd.0002097

7. Hayman DT, Banyard AC, Wakeley PR, Harkess G, Marston D, Wood JL, et al. A universal real-time assay for the detection of lyssaviruses. J Virol Methods. 2011;177:87-93. 10.1016/j.jviromet. 2011.07.002 http://dx.doi.org/10.1016/j.jviromet.2011.07.002

8. Franka R, Constantine DG, Kuzmin I, Velasco-Villa A, Reeder SA, Streicker D, et al. A new phylogenetic lineage of rabies virus associated with western pipistrelle bats (Pipistrellus hesperus). J Gen Virol. 2006;87:2309-21. http://dx.doi.org/10.1099/ vir.0.81822-0

9. Trimarchi CV, Smith JS. Diagnostic evaluation. In: Press A, Jackson AC, Wunner WH, editors. Rabies. 1st ed. San Diego (CA): Academic Press; 2002. p. 308-44.

10. Mayer F, von Helversen O. Cryptic diversity in European bats. Proc Biol Sci. 2001;268:1825-32. http://dx.doi.org/10.1098/ rspb.2001.1744

11. Bozzola JJ, Russell LD. Electron microscopy: principles and techniques for biologists. Burlington (MA): Jones \& Bartlett Learning; 1992. p. 130-134.

12. World Health Organization. WHO Expert Consultation on Rabies. Second report. World Health Organ Tech Rep Ser. 2013;982:1-139.

13. Francis CM. Chiroptera In: Mayer K, editor. A field guide to the mammals of South-East Asia, 1st ed. London: New Holland; 2008. p. 238.

14. Srinivasulu B, Srinivasulu C, Kaur H, Srinivasulu A. A new distribution record of the Japanese pipistrelle (Pipistrellus abramus (Temminck, 1840); Mammalia, Chiroptera) in India. Acta Zoologica Lituanica. 2011;21:268-72. http://dx.doi.org/10.2478/ v10043-011-0029-8

Address for correspondence: Wei-Cheng Hsu, Animal Health

Research Institute, Council of Agriculture, Executive Yuan, No. 376, Chung-Cheng Rd., Tamsui District, New Taipei City 25158, Taiwan; email:wchsu@mail.nvri.gov.tw

\section{EID Podcast: Bat Flight and Zoonotic Viruses}

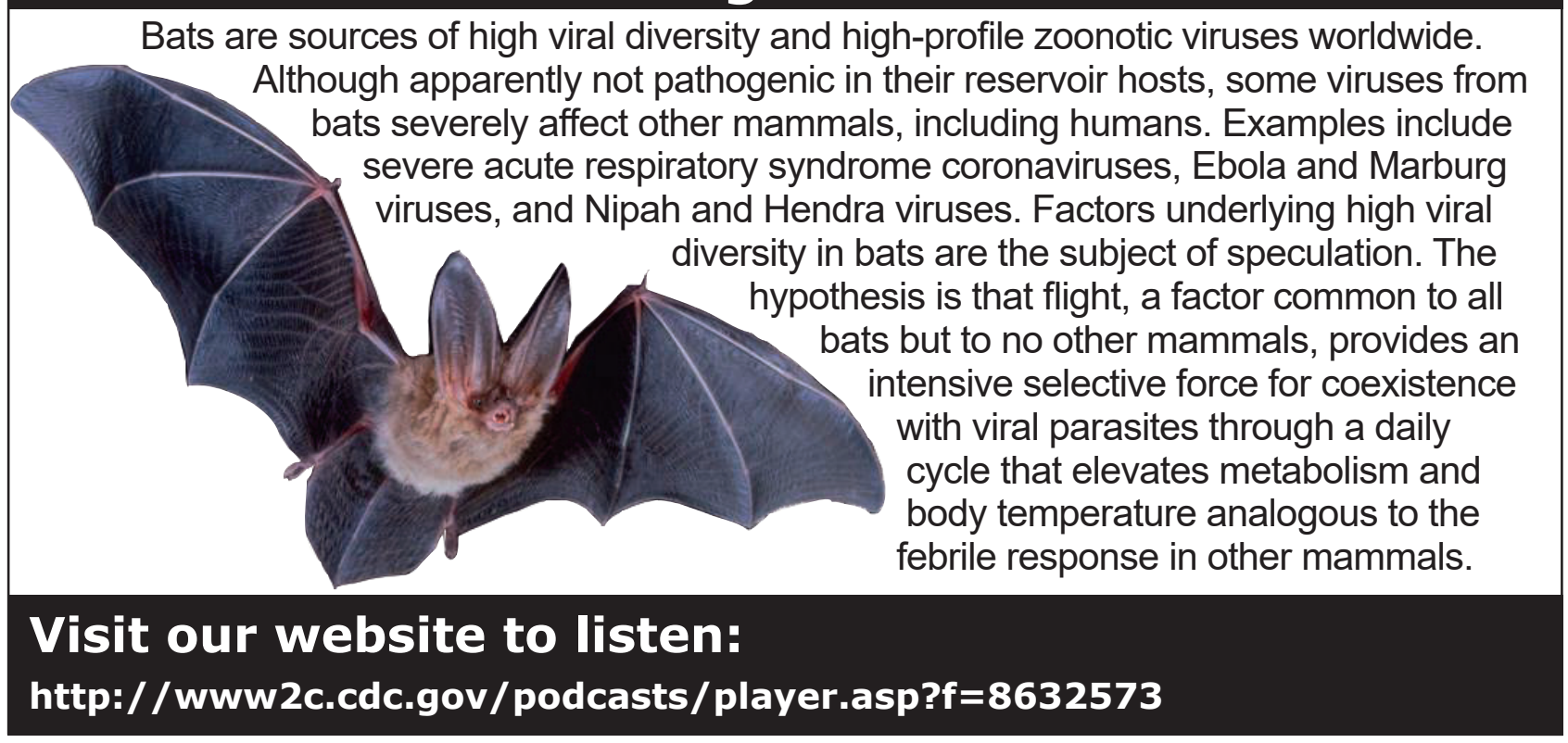

